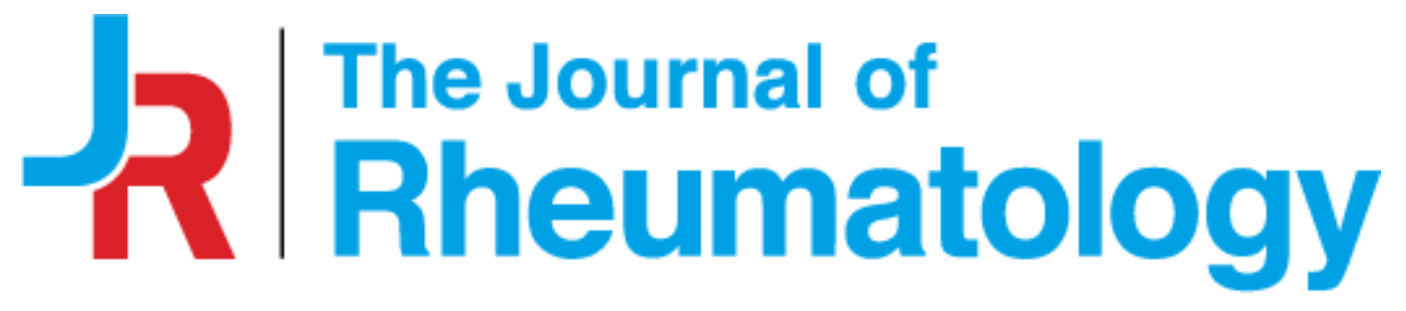

The Journal of Rheumatology

Volume 40, no. 1

Safety and Efficacy of Infliximab and Adalimumab for Refractory Uveitis in Juvenile Idiopathic Arthritis: 1-year Followup Data from the Italian Registry

MARIA E. ZANNIN, CAROLINA BIROLO, VALERIA M. GERLONI, ELISABETTA MISEROCCHI, IRENE PONTIKAKI, MARIA P. PAROLI, CLAUDIA BRACAGLIA, ALISON SHARDLOW, FULVIO PARENTIN, ROLANDO CIMAZ, GABRIELE SIMONINI, FERNANDA FALCINI, FABRIZIA CORONA, STEFANIA VIOLA, RICCARDO DE MARCO, LUCIANA BREDA, FRANCESCO LA TORRE, FABIO VITTADELLO, GIORGIA MARTINI and FRANCESCO ZULIAN

J Rheumatol 2013;40;74-79

http://www.jrheum.org/content/40/1/74

1. Sign up for TOCs and other alerts

http://www.jrheum.org/alerts

2. Information on Subscriptions

http://jrheum.com/faq

3. Information on permissions/orders of reprints

http://jrheum.com/reprints_permissions

The Journal of Rheumatology is a monthly international serial edited by Earl D.

Silverman featuring research articles on clinical subjects from scientists working in rheumatology and related fields. 


\title{
Safety and Efficacy of Infliximab and Adalimumab for Refractory Uveitis in Juvenile Idiopathic Arthritis: 1-year Followup Data from the Italian Registry
}

\author{
MARIA E. ZANNIN, CAROLINA BIROLO, VALERIA M. GERLONI, ELISABETTA MISEROCCHI, \\ IRENE PONTIKAKI, MARIA P. PAROLI, CLAUDIA BRACAGLIA, ALISON SHARDLOW, FULVIO PARENTIN, \\ ROLANDO CIMAZ, GABRIELE SIMONINI, FERNANDA FALCINI, FABRIZIA CORONA, STEFANIA VIOLA, \\ RICCARDO DE MARCO, LUCIANA BREDA, FRANCESCO LA TORRE, FABIO VITTADELLO, GIORGIA MARTINI, \\ and FRANCESCO ZULIAN
}

ABSTRACT. Objective. To evaluate safety and efficacy of adalimumab (ADA) and infliximab (IFX) for the treatment of juvenile idiopathic arthritis-related anterior uveitis (JIA-AU).

Methods. Starting January 2007, patients with JIA-AU treated with IFX and ADA were managed by a standard protocol and data were entered into the National Italian Registry (NIR). At baseline, all patients were refractory to standard immunosuppressive treatment and/or were corticosteroid-dependent. Data recorded every 3 months included uveitis course, number/type of ocular complications, drug-related adverse events (AE), treatment change or withdrawal, and laboratory measures. Data of patients treated for at least 1 year were retrieved from the NIR and analyzed using descriptive statistics. Treatment efficacy was based on change in uveitis course and in number of ocular complications.

Results. Up to December 2009, data for 108 patients with JIA-AU treated with anti-tumor necrosis factor- $\square$ agents were recorded in the NIR and data from 91, with at least 12 months' followup, were included in the study. Forty-eight patients were treated with IFX, 43 with ADA. Forty-seven patients (55.3\%) achieved remission of AU, 28 (32.9\%) had recurrent AU, and 10 (11.8\%) maintained a chronic course. A higher remission rate was observed with ADA $(67.4 \%$ vs $42.8 \%$ with IFX; $\mathrm{p}=$ $0.025)$. Ocular complications decreased from 0.47 to 0.32 per subject. Five patients experienced resolution of structural complications. No patient reported serious AE; $8(8.8 \%)$ experienced 11 minor AE (9 with IFX, 2 with ADA).

Conclusion. IFX and ADA appear to be effective and safe for treatment of refractory JIA-related uveitis, with a better performance of ADA in the medium-term period. (First Release Nov 12012 ; J Rheumatol 2013;40:74-9; doi:10.3899/jrheum.120583)

Key Indexing Terms:

UVEITIS

ANTI-TUMOR NECROSIS FACTOR AGENTS

JUVENILE IDIOPATHIC ARTHRITIS TREATMENT
From the Ophthalmology and Rheumatology Units, Department of Pediatrics, University of Padua, University of Milan (G. Pini Institute), University of Rome (Ocular Immunovirology Service, Sapienza University), University of Rome (Bambino Gesù Children's Hospital), University of Trieste (Burlo Garofalo Children's Hospital), University of Florence (A. Meyer Children's Hospital), University of Florence (Department of Internal Medicine, Rheumatology, Transition Clinic), University of Milan (Fondazione IRCCS Cà Granda-Ospedale Maggiore Policlinico), University of Genoa (G. Gaslini Children's Hospital), University of Chieti (University Hospital), and University of Messina (University Hospital), Italy.

Supported by a dedicated grant from Il Volo, Association for Rheumatic Diseases in Children, Onlus, Padua, Italy.

F. Zulian, MD, Professor OC, Rheumatology Unit; M.E. Zannin, MD, PhD; C. Birolo, MD, Assistant Professor, Ophthalmology Unit; G. Martini, MD, Assistant Professor; F. Vittadello, Statistician, Rheumatology Unit, Department of Pediatrics, University of Padua; V. Gerloni, MD, Associate Professor; I. Pontikaki, MD, Assistant Professor, Rheumatology Unit; E. Miserocchi, MD, Assistant Professor,

\author{
Ophthalmology Unit, Pediatric Rheumatology Center, G. Pini Hospital; \\ C. Bracaglia, MD, Assistant Professor, Bambino Gesù Children's \\ Hospital; M.P. Paroli, MD, Assistant Professor, Ocular Immunovirology \\ Service, Sapienza University; A. Shardlow, MD, Assistant Professor, \\ Rheumatology Unit; F. Parentin, MD, Assistant Professor, Ophthalmology \\ Unit, Burlo Garofalo Children's Hospital; R. Cimaz, MD, Associate \\ Professor; G. Simonini, MD, Assistant Professor, Rheumatology Unit, A. \\ Meyer Children's Hospital; F. Falcini, MD, Associate Professor, \\ Rheumatology Unit, Transition Clinic, University of Florence; F. Corona, \\ MD, Assistant Professor, Fondazione IRCCS Cà Granda-Ospedale \\ Maggiore Policlinico; S. Viola, MD, Assistant Professor, Rheumatology \\ Unit; R. De Marco, MD, Assistant Professor, Ophthalmology Unit, G. \\ Gaslini Children's Hospital; L. Breda, MD, Assistant Professor, Chieti \\ University Hospital; F. La Torre, MD, Assistant Professor, Messina \\ University Hospital. \\ Address correspondence to Dr. M.E. Zannin, MD, Department of \\ Pediatrics, University of Padua, Via Giustiniani 3, 35128 Padua, Italy. \\ E-mail:ezannin@pediatria.unipd.it \\ Accepted for publication September 7, 2012.
}


Anterior uveitis (AU) is the most important extraarticular complication of juvenile idiopathic arthritis (JIA) and may lead to severe visual impairment due to sight-threatening complications such as posterior synechiae, band keratopathy, cataract, and glaucoma ${ }^{1}$. Although treatment of AU is not standardized, it is increasingly evident that early aggressive therapy is needed to prevent irreversible complications and preserve visual function.

The introduction of anti-tumor necrosis factor- $\alpha$ (anti-TNF- $\alpha$ ) agents has significantly changed the management of many rheumatic conditions, including AU, but experience to date is still limited. Since January 2007 the Italian National Agency for Drugs has approved the use of the anti-TNF- $\alpha$ agents infliximab (IFX) and adalimumab (ADA) as off-label treatment for refractory JIA-related uveitis. Because of the lack of information on the longterm safety and efficacy of these new agents, a National Italian Registry (NIR) was established and data were prospectively collected to periodically update the health authorities. We report the data retrieved from the registry on the safety and efficacy of IFX and ADA in patients treated for at least 1 year.

\section{MATERIALS AND METHODS}

Patients with JIA-related uveitis treated with the anti-TNF- $\alpha$ agents IFX and ADA and followed since January 2007 at pediatric rheumatology and ophthalmologic institutions in Italy were managed by a standardized protocol and data were prospectively collected in the NIR. All patients were diagnosed as having JIA according to the International League of Associations for Rheumatology criteria ${ }^{2}$ and AU according to the SUN Working Group criteria ${ }^{3}$.

For treatment, all participating centers adopted a "stepladder" algorithm. New-onset uveitis was treated just with mydriatics and/or topical corticosteroid therapy. In case of recurrent or persistent AU, a brief course (1-2 months) of systemic corticosteroids (oral prednisone $0.5-1$ $\mathrm{mg} / \mathrm{kg} /$ day) was prescribed. Immunosuppressive treatment [methotrexate (MTX) $10-15 \mathrm{mg} / \mathrm{m}^{2} /$ week or cyclosporine A (CYA) $3-5 \mathrm{mg} / \mathrm{kg} / \mathrm{day}$ ] was used in cases of refractory or steroid-dependent AU. Finally, anti-TNF agents were considered for patients refractory to MTX or CYA.

The indications to start anti-TNF treatment were intolerance or nonresponsiveness to at least 6 months of MTX and/or CYA treatment, and/or oral prednisone dependency.

The anti-TNF- $\alpha$ agents IFX or ADA were administered according to the Italian National Health System regulations. Intravenous IFX infusions, dosage $5 \mathrm{mg} / \mathrm{kg}$, were scheduled at $0,2,6$, and 12 weeks and then continued every 6-8 weeks; ADA was administered subcutaneously every 2 weeks at a dosage of $1 \mathrm{mg} / \mathrm{kg}$ (maximum $40 \mathrm{mg}$ ). In patients refractory to treatment, shorter IFX infusion intervals were tried (maximum every 4 weeks). In patients receiving ADA, the dose was progressively increased to a maximum $40 \mathrm{mg}$.

Consecutive ophthalmologic evaluations were performed at at least 3-month intervals. Data collected were uveitis disease course, number and type of ocular complications, drug-related adverse events (AE), causes of treatment change or withdrawal, and laboratory measurements. Concomitant use of topical and systemic corticosteroids and systemic immunosuppressive agents was documented. Sparing of topical and systemic corticosteroid and immunosuppressive drugs during anti-TNF treatment was recorded.

The uveitis disease course was defined according to the SUN Working Group criteria ${ }^{3}$. Chronic (severe) uveitis was considered the presence of persistent or unremitting uveitis or a uveitis relapse every 3 months or less, or the presence of at least 3 relapses/year. Recurrent (moderate) uveitis was considered when repeated flares of AU were separated by periods of inactivity longer than 3 months' duration or when there were fewer than 3 relapses/year.

Clinical remission on medication was defined as the absence of active uveitis for more than 6 months on systemic treatment and with no or minimal topical treatment (corticosteroid and/or mydriatic-cycloplegic eyedrops used less than once daily).

Uveitis flare was defined as an increase of the cells in the anterior chamber of $2+$ or more compared to baseline ${ }^{3}$. Ocular complications were recorded at baseline, at start of treatment, and then during each followup visit. Ocular hypertension was diagnosed in patients in whom the intraocular pressure (IOP) had been $>22 \mathrm{~mm} \mathrm{Hg}$ for $>3$ months, or a single IOP measurement was $>30 \mathrm{~mm} \mathrm{Hg}^{4}$.

Patients were monitored for $\mathrm{AE}$, vital signs, and laboratory assessments at each study visit. Serious AE, defined as those requiring hospitalization, and changes in vital signs for a minimum of $1 \mathrm{~h}$ from the start of each anti-TNF administration, were recorded. As recommended, Quantiferon TB Gold test screening was performed before initiation of the treatment TNF inhibitors and at least yearly during the followup ${ }^{5}$.

Laboratory monitoring included white blood cell (WBC) count, hemoglobin, platelet count, erythrocyte sedimentation rate (ESR), C-reactive protein (CRP), alanine transaminase (ALT), aspartate transaminase (AST), creatinine, blood urea nitrogen (BUN), and urinalysis. ESR and CRP were considered abnormal if $>35 \mathrm{~mm} / \mathrm{h}$ and $0.5 \mathrm{mg} / \mathrm{dl}$, respectively. WBC counts and hemoglobin were considered abnormal if greater or less than $2 \mathrm{SD}$ values for age. Platelet count was considered abnormal if $>$ $400 \times 10^{3} / \mathrm{mm}^{3}$ or $<150 \times 10^{3} / \mathrm{mm}^{3}$. ALT and AST were considered abnormal if $>40 \mathrm{U} / \mathrm{l}$, creatinine if $>62 \mu \mathrm{mol} / 1$ or $0.47 \mathrm{mg} / \mathrm{dl}$, and BUN if $>6.40 \mathrm{mmol} / \mathrm{l}$. Antinuclear antibodies, tested by immunofluorescence on Hep2 cell line, were considered positive with a titer $>1: 80$. Anti-dsDNA and extractable nuclear antigen were considered positive or abnormal according to the standard value of the laboratory of each participating center. Tests were performed at baseline and then every 3 months during the followup and were concomitant with the clinical assessment. Any treatment change or withdrawal was also recorded.

Statistical analysis. Data for patients treated with anti-TNF agents for at least 1 year were retrieved anonymously from the NIR and analyzed by descriptive statistics. A comparative analysis on safety and efficacy of IFX and ADA was performed. Patients undergoing a treatment change from IFX to ADA or vice versa were excluded from the efficacy analysis but included in that for safety. All significance tests were 2-sided and $\mathrm{p}$ values $<0.05$ were considered significant. All analyses were performed using StatsDirect (version 2.7.5, StatsDirect Ltd.).

\section{RESULTS}

During the period January 2007-December 2009, 108 patients with JIA-AU were included in the NIR, and 91 (71 female, 20 male) with at least 12 months of followup entered our study. Clinical characteristics of the patients are summarized in Table 1. The mean age at start of treatment was 9.7 years and the duration of uveitis was 4.9 years. The mean followup duration after treatment start was 1.5 years (range 1-3 yrs). The mean age at arthritis onset was 4.5 years, while the mean age at uveitis onset was 4.9 years. According to the diagnostic criteria for JIA of the International League of Associations for Rheumatology ${ }^{2}$, 90.2\% had persistent and 9.8\% extended oligoarticular JIA. All patients had failed previous immunosuppressive treatments (MTX, CYA) and/or were dependent on oral corticosteroids. 
Table 1. Baseline demographic and clinical characteristics of the patients. Data are number $(\%)$ or mean (SD) unless otherwise indicated.

\begin{tabular}{lcc}
\hline Characteristic & $\begin{array}{c}\text { Adalimumab, } \\
\mathrm{n}=43\end{array}$ & $\begin{array}{c}\text { Infliximab, } \\
\mathrm{n}=48\end{array}$ \\
\hline Sex, F:M & $5.1: 1$ & $2.7: 1$ \\
Age, yrs & $8.8(4.4)$ & $10.5(4.3)$ \\
Age at arthritis onset, yrs & $3.9(3.2)$ & $4.8(3.2)$ \\
$\quad$ Range & $1.0-15.6$ & $0.6-13.4$ \\
Age at uveitis onset, yrs & $4.7(4.2)$ & $4.9(3.3)$ \\
Range & $1.0-18.0$ & $0.9-15.0$ \\
Time interval arthritis/uveitis, mo & $18.5(24.9)$ & $27.2(33.1)$ \\
$\quad$ Range & $0.0-120.1$ & $0.0-137.7$ \\
Uveitis duration, yrs & $4.1(3.5)$ & $5.5(4.7)$ \\
$\quad$ Range & $0.0-15.9$ & $0.3-18.0$ \\
Previous treatments & & $41(85.4)$ \\
$\quad$ Methotrexate & $35 *(81.4)$ & $10(20.8)$ \\
Cyclosporine A & $3(7.0)$ & $18(37.5)$ \\
Corticosteroid dependency & $17(39.5)$ &
\end{tabular}

* Five patients in this group were intolerant to methotrexate and were not considered because of the very short $(<3$ weeks) treatment duration.

Forty-three patients were initially treated with ADA, 48 with IFX. Comparison of the 2 groups at baseline showed no significant differences (Table 1). Seventy-five patients $(83.5 \%)$ were receiving MTX at baseline and 58 (63.7\%) continued the treatment afterward $(\mathrm{p}<0.001)$. CYA-treated patients also decreased in number, from $13(14.3 \%)$ at baseline to $7(7.7 \%)$ during anti-TNF therapy $(\mathrm{p}<0.05)$. Of 35 patients $(38.5 \%)$ taking prednisone at baseline, 16 $(17.6 \%)$ were able to discontinue it during the study period. Indeed, for those who were still taking immunosuppressive treatment at the end of the yearly observation period, the mean dosage of drugs could be gradually tapered as follows: MTX from 10.2 to $8.8 \mathrm{mg} / \mathrm{m}^{2} /$ week, CYA from 3.4 to 2.6 $\mathrm{mg} / \mathrm{kg} / \mathrm{day}$, and prednisone from 0.52 to $0.11 \mathrm{mg} / \mathrm{kg} / \mathrm{day}$ (mean values). Topical corticosteroid eyedrops could be discontinued in 38 patients and tapered in 53. None of the patients received subtenon or intravitreal corticosteroid injection.

During the first year of treatment, 6 patients $(6.6 \%)$, all receiving IFX, changed therapy: in 3, IFX was interrupted because of infusion reactions, in the remainder because of diminished tolerance or compliance. All were shifted to ADA, after a mean 7.4 months (range 3.3-10.3 mo) from the start of treatment. No patient changed from ADA to IFX.

For the course of uveitis, 47 patients $(55.3 \%)$ achieved remission, 28 (32.9\%) had recurrent uveitis, and 10 (11.8\%) maintained a chronic disease course (Table 2). A significant difference between IFX and ADA treatment was observed, with a better remission rate for ADA $(67.4 \%$ vs $42.8 \%$ for IFX; $\mathrm{p}=0.025$ ). Among 29 patients with a recurrent uveitis course at treatment start, $17(58.6 \%)$ achieved remission and $12(41.4 \%)$ remained stable. Among 56 patients with a chronic uveitis course at baseline, $82.2 \%$ improved significantly: 30 patients $(53.6 \%)$ achieved remission, $16(28.6 \%)$
Table 2. Change of uveitis course after treatment with anti-tumor necrosis factor agents. Data are number (\%) unless otherwise indicated.

\begin{tabular}{lccc}
\hline & \multicolumn{2}{c}{ Posttreatment Uveitis Course } \\
Pretreatment Uveitis Course & Chronic & Recurrent & Remission \\
\hline Adalimumab, $\mathrm{n}=43$ & & & \\
Chronic, $\mathrm{n}=26$ & $3(11.5)$ & $6(23.1)$ & $17(65.4)$ \\
Recurrent, $\mathrm{n}=17$ & $0(0.0)$ & $5(29.4)$ & $12(70.6)$ \\
Overall & $3(7)$ & $11(25.6)$ & $29(67.4)^{\dagger}$ \\
Infliximab, $\mathrm{n}=42$ & & & \\
Chronic, $\mathrm{n}=30$ & $7(23.3)$ & $10(33.3)$ & $13(43.3)$ \\
Recurrent, $\mathrm{n}=12$ & $0(0.0)$ & $7(58.3)$ & $5(41.7)$ \\
Overall & $7(16.7)$ & $17(40.5)$ & $18(42.8)^{\dagger}$ \\
Overall, $\mathrm{n}=85$ & & & \\
Chronic, $\mathrm{n}=56$ & $10(17.8)$ & $16(28.6)$ & $30(53.6)$ \\
Recurrent, $\mathrm{n}=29$ & $0(0.0)$ & $12(41.4)$ & $17(58.6)$ \\
Overall & $10(11.8)$ & $28(32.9)$ & $47(55.3)$ \\
\hline p $<0.05$. & & &
\end{tabular}

had a reduced rate of uveitis flares (recurrent course), and only $10(17.8 \%)$ maintained a chronic disease course.

At treatment start, 23 patients (27.1\%) showed 40 ocular complications (Figure 1), which were multiple in 12 $(14.1 \%)$. Cataract was present in 17 subjects (20.0\%), vitreitis in $12(14.1 \%)$, cystoid macular edema [CME, confirmed by optical coherence tomography (OCT)] in 6 $(7.1 \%)$, and ocular hypertension in $5(5.9 \%)$. As absolute values, complications decreased from 40 ( 0.47 per subject) to 27 (0.32 per subject), with $32 \%$ improvement. Five patients experienced complete resolution of structural complications. In 2 of them it was achieved in combination with a cataract surgery procedure. In particular, the cataract surgery was possible only because of the prior achievement of remission of inflammation due to anti-TNF treatment. Therefore, the successful result should be attributed to both medical and surgical interventions. Of 62 patients without complications at treatment start, only $3(4.8 \%)$ developed a new cataract. Two of them were receiving IFX, and 1 ADA. Considering changes of structural complications, no significant difference between the 2 anti-TNF- $\alpha$ agents was observed.

During treatment, no patient reported serious or life-threatening AE. Eight (8.8\%) experienced 11 minor AE (12.1 per 100 patient/yrs; Table 3). Nine AE were related to IFX treatment (18.8 per 100 patient/yrs) and 2 to ADA (4.7 per 100 patient/yrs).

No significant laboratory changes related to anti-TNF agents were observed and no significant differences were found comparing IFX and ADA. Analysis of autoantibodies showed that the percentage of patients with positive antinuclear antibody tests decreased from $29.7 \%$ to $14.3 \%$ during followup, while 3 subjects were found to have positive anti-dsDNA antibody results at low titer. Two were receiving IFX and 1 ADA. However, these findings were not associated with features of lupus-like syndrome. 


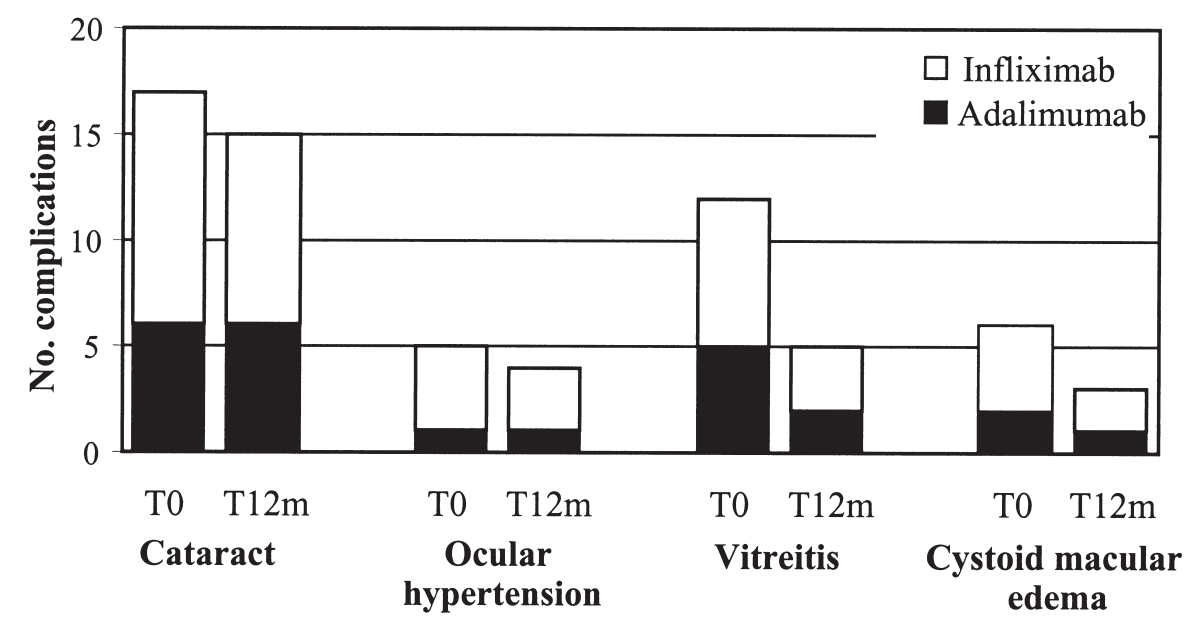

Figure 1. Ocular complications at baseline and after 12 months of treatment with infliximab or adalimumab.

Table 3. Adverse events (AE) related to anti-tumor necrosis factor treatment. Data are number $(\%)$ unless otherwise indicated.

\begin{tabular}{lcc}
\hline Adverse Event & Infliximab, $\mathrm{n}=48$ & Adalimumab, $\mathrm{n}=43$ \\
\hline Major AE & 0 & 0 \\
Minor AE & $9(18.7)$ & $2(4.7)$ \\
Infections & 3 & 0 \\
Upper respiratory tract & 1 & 0 \\
Herpes zoster virus & 1 & 0 \\
Urinary tract & 1 & 0 \\
Infusion reactions & 3 & 0 \\
Systemic symptoms & 3 & 2 \\
Headache & 2 & 0 \\
Irritability & 1 & 0 \\
Urticarial rash & 0 & 1 \\
Prolonged menses & 0 & 1 \\
\hline
\end{tabular}

\section{DISCUSSION}

The treatment of JIA-AU varies considerably in type and timing and frequently does not adequately control intraocular inflammation; side effects and poor visual outcome are still quite common $6,7,8,9$. Anti-TNF- $\alpha$ agents have recently been indicated as possible therapeutic options to treat AU and to prevent ocular complications ${ }^{10,11,12}$.

We report the safety and efficacy results of the first year of anti-TNF- $\alpha$ treatment in a large cohort of patients with refractory JIA-AU with data recorded in the NIR. This cohort represents the largest and most homogeneous group of pediatric patients with AU treated with anti-TNF- $\alpha$ reported to date. It includes data for patients with oligoarticular JIA, the subtype more prone to develop refractory uveitis. Other subtypes, such as polyarticular, psoriatic, and enthesitis-related JIA, are rarely affected by severe-course uveitis by themselves, probably because they receive TNF-blocker treatment early in the disease course to control the arthritis.
At the start of anti-TNF- $\alpha$ treatment, one-third of the patients already had a complicated uveitis and as many as two-thirds had a chronic (severe) uveitis course. To assess the efficacy of anti-TNF- $\alpha$ agents, we analyzed changes in the uveitis course in terms of number of uveitis flares compared with the previous year, and changes in ocular complications.

Both IFX and ADA were found to be effective in JIA-AU, as $88.2 \%$ of the patients improved; half achieved remission and one-third maintained a recurrent course. Indeed, the rate of chronic-severe disease course dropped from $65.9 \%$ to $11.8 \%$. These results confirm some previous retrospective studies in which ADA was effective in reducing the ocular inflammation and the recurrence rate in the majority of JIA patients with longstanding uveitis, which was poorly responsive to conventional treatments ${ }^{13,14}$.

Interestingly, in our study the comparison between ADA and IFX showed better results for ADA, in that the overall remission rate was $67.4 \%$, versus $42.8 \%$ with IFX ( $p=$ $0.025)$. This result is in contrast with another study showing no difference in the uveitis remission rate between the 2 treatment regimens ${ }^{15}$, and it demonstrates that ADA is more efficacious in maintaining remission of uveitis over time. That study ${ }^{15}$, however, was different from ours in that it included a smaller, heterogeneous patient cohort with different types of uveitis, such as idiopathic, Behçet, sarcoid, and JIA-related uveitis. These different features partly explain the diverging results of the 2 studies.

On treatment with anti-TNF agents, ocular complications improved significantly, as their number decreased from 0.47 to 0.32 per subject. Only 3 patients ( 2 on IFX and 1 ADA) experienced new-onset cataract, but as suggested by other authors, this complication was probably due to previous prolonged topical corticosteroid treatment ${ }^{16,17}$. In a retrospective case series of 24 children treated with anti-TNF- $\alpha$ 
agents, a significant rate $(17 \%)$ of worsening of preexisting glaucoma occurred during IFX treatment ${ }^{7}$. In general, while the improvement in number and severity of some ocular complications, such as glaucoma or cataract, is usually related to treatment, worsening can be a reflection of existing damage from previous inflammation or steroid treatment and is not necessarily a result of ongoing inflammation.

Of note, the frequency of vitreitis and CME decreased significantly during the first year of treatment. Similar results have been reported in a series of 19 patients, including adults, treated with $\mathrm{ADA}$ for 1 year ${ }^{18}$. In that study, CME (detected by OCT in $86 \%$ of the eyes at baseline) resolved completely in $54.5 \%$ of subjects. Similarly, we observed resolution of CME in $50 \%$ of the patients in our study, although this complication was present in only 6 .

Recent studies provide preliminary evidence that, in refractory uveitis with a loss of initial clinical response to a biologic agent, switching to another agent can restore the control of intraocular inflammation ${ }^{19,20}$. In a recent case series, 7 patients with refractory uveitis switched therapy from IFX to ADA and achieved remission ${ }^{19}$. Indeed, this change may offer several adjunctive advantages, such as easier administration, better patient compliance, and lower rate of $\mathrm{AE}^{20}$. In our study, 6 patients switched from IFX to ADA because of infusion reactions, intolerance, or difficult compliance. However, because of the short followup after switching, it was not possible to evaluate the real significance of this change. The safety profile of both anti-TNF- $\alpha$ agents was acceptable, as only $8.8 \%$ developed minor AE. ADA was better tolerated, as only 1 case of transient urticarial rash and 1 of prolonged menses were observed. Conversely, a study of 26 patients with JIA who were treated with ADA for arthritis reported a higher rate of AE (26.9\%), mostly respiratory tract infections ${ }^{21}$. Also in contrast with previous studies ${ }^{14}$, we were surprised that local reactions to ADA were reported rarely by our patients. The reason for this could be 2-fold; in most of the patients, ADA injection was preceded by the application of anesthetic cream (EMLA). Second, the high motivation to perform the treatment for a risky complication and the high expectation from the parents may have reduced the psychological effect of possible local reactions, which were probably reported less frequently.

Almost one-fifth of our patients receiving IFX developed 1 or more AE, which included infections (3 cases), flu-like symptoms (3 cases), and infusion reactions (3 cases). Similarly, a retrospective study on a series of 20 children showed a comparable rate of AE (15\%), mostly infections and infusion reactions ${ }^{22}$.

The autoantibody testing revealed anti-DNA antibodies in 3 patients ( 2 on IFX, 1 ADA), but no lupus-like symptoms were reported. This confirms data from a recent study that autoantibody screening tests in pediatric patients treated with anti-TNF agents were mostly negative or nonspecific, suggesting a less frequent screening program ${ }^{23}$.

Data from the NIR show that IFX and ADA appear to be effective and safe for treatment of refractory JIA-related uveitis, with a better performance of ADA in the medium term. Continuing collection of data from this registry, involving pediatric rheumatologists and ophthalmologists, will provide further information on the longterm efficacy and safety of these and other biologic agents.

\section{ACKNOWLEDGMENT}

We thank A. Andreotti for statistical support. We are grateful to the following physicians for their participation in the National Italian Registry; Rheumatologists: M. Alessio, Naples; A. Ravelli and M.G. Alpigiani, Genoa; R. Barcellona, Palermo; P. Barone and R. Garozzo, Catania; D. Rigante, E. Cortis, and F. De Benedetti, Rome; A. De Fanti, Reggio Emilia; A. Hila, Milan; M. Jorini, Ancona; L. Lepore, Trieste; S. Martino, Turin; A. Miniaci and A. Pession, Bologna; P. Sacchini, Rimini; and M. Sprocati and C. Host, Ferrara. Ophthalmologists: R. Caputo and C. De Libero, Florence; L. Cimino, Reggio Emilia; F. Gnocchini, Ancona; L. Latanza, Naples; P. Monari, Ferrara; A. Brancaleoni, M. Ortolani, and F. Pietropaolo, Rimini; C. Pirondini, Chieti; and A. Romanzo, Rome.

\section{REFERENCES}

1. Thorne JE, Woreta F, Kedhar SR, Dunn JP, Jabs DA. Juvenile idiopathic arthritis associated uveitis: Incidence of ocular complications and visual acuity loss. Am J Ophthalmol 2007;143:647-55.

2. Petty RE, Southwood TR, Manners P, Baum J, Glass DN, Goldenberg $\mathrm{J}$, et al. International League of Associations for Rheumatology classification of juvenile idiopathic arthritis: second revision, Edmonton, 2001. J Rheumatol 2004;31:390-2.

3. Jabs DA, Nussenblatt RB, Rosenbaum JT. Standardization of uveitis nomenclature for reporting clinical data. Result of the First International Workshop. J Ophthalmol 2005;140:509-16.

4. Kotaniemi K, Sihto-Kauppi K. Occurrence and management of ocular hypertension and secondary glaucoma in juvenile idiopathic arthritis-associated uveitis: An observational series of 104 patients. Clin Ophthalmol 2007;1:455-9.

5. Rosenblum H, Amital H. Anti-TNF therapy: Safety aspects of taking the risk. Autoimmun Rev 2011;10:563-8.

6. Kump LI, Castaneda RA, Andreoudi SN, Reed GF, Foster CS. Visual outcomes in children with juvenile idiopathic arthritis-associated uveitis. Ophthalmology 2006;113:1874-7.

7. Saurenmann RK, Levin AV, Rose JB, Parker S, Rabinovitch T, Tyrrell PN, et al. Tumour necrosis factor alpha inhibitors in the treatment of childhood uveitis. Rheumatology 2006;45:982-9.

8. Woreta F, Thorne JE, Jabs DA, Kedhar SR, Dunn JP. Risk factors for ocular complications and poor visual acuity at presentation among patients with uveitis associated with juvenile idiopathic arthritis. Am J Ophthalmol 2007;143:647-55.

9. Bolt IB, Cannizzaro E, Seger R, Saurenmann RK. Risk factors and long-term outcome of juvenile idiopathic arthritis-associated uveitis in Switzerland. J Rheumatol 2008;35:703-6.

10. Theodossiadis PG, Markomichelakis NN, Sfikakis PP. Tumor necrosis factor antagonists: preliminary evidence for an emerging approach in the treatment of ocular inflammation. Retina 2007;27:399-413.

11. Richards JC, Tay-Kearney ML, Murray K, Manners P. Infliximab for juvenile idiopathic arthritis associated uveitis. Clin Exp Ophthalmol 2005;35:461-8. 
12. Tynjala P, Kotaniemi K, Lindahl P, Latva K, Aalto K, Honkanen V. Adalimumab in juvenile idiopathic arthritis associated chronic anterior uveitis. Rheumatology 2008;47:339-44.

13. Biester S, Deuter C, Michels H, Haefner R, Kuemmerle-Deschner $\mathrm{J}$, Doycheva D, et al. Adalimumab in the therapy of uveitis in childhood. Br J Ophthalmol 2007;91:319-24.

14. Vasquez-Cobian LB, Flynn T, Lehman TJ. Adalimumab therapy for childhood uveitis. J Pediatr 2006;149:572-5.

15. Simonini G, Taddio A, Cattalini M, Caputo R, De Libero C, Naviglio S, et al. Prevention of flare recurrences in childhood refractory chronic uveitis: An open-label comparative study of adalimumab versus infliximab. Arthritis Care Res 2011;63:612-8.

16. Jobling AI, Augusteyn RC. What causes steroid cataracts? A review of steroid-induced posterior subcapsular cataracts. Clin Exp Optom 2002;85:61-75.

17. James ER. The etiology of steroid cataract. J Ocul Pharmacol Ther 2007;23:403-20.

18. Diaz-Llopis M, Garcia-Delpech S, Salom D, Udaondo P, Hernández-Garfella M, Bosch-Morell F, et al. Adalimumab therapy for refractory uveitis: A pilot study. J Ocul Pharmacol Ther 2008;24:351-61.
19. Dhingra N, Morgan J, Dick AD. Switching biologic agents for uveitis. Eye 2009;23:1868-70.

20. Neri P, Zucchi M, Allegri P, Lettieri M, Mariotti C, Giovannini A. Adalimumab (Humira): A promising monoclonal anti-tumor necrosis factor alpha in ophthalmology. Int Ophthalmol 2011;31:165-73.

21. Trachana M, Pratsidou-Gertsi P, Pardalos G, Kozeis N, Badouraki M, Kanakoudi-Tsakalidou F. Safety and efficacy of adalimumab treatment in Greek children with juvenile idiopathic arthritis. Scand J Rheumatol 2011;40:101-7.

22. Tugal-Tutkun I, Ayranci O, Kasapcopur O, Kir N. Retrospective analysis of children with uveitis treated with infliximab. J AAPOS 2008;12:611-3.

23. Duncan J, Heath J, Baildam E, Cleary G, Beresford M, McCann LJ. Autoantibody testing for children on biologic therapies for rheumatologic conditions: Result of audit [abstract]. Pediatr Rheum 2011;9:81-2. 


\section{Correction}

\section{Safety and Efficacy of Infliximab and Adalimumab for Refractory Uveitis in Juvenile Idiopathic Arthritis: 1-year Followup Data from the Italian Registry}

Zannin ME, Birolo C, Gerloni VM, Miserocchi E, Pontikaki I, Paroli MP, Bracaglia C, Shardlow A, Parentin F, Cimaz R, Simonini G, Falcini F, Corona F, Viola S, De Marco R, Breda L, La Torre F, Vittadello F, Martini G, Zulian F. Safety and efficacy of infliximab and adalimumab for refractory uveitis in juvenile idiopathic arthritis: 1-year followup data from the Italian Registry. J Rheumatol 2013;40:74-9. The correct affiliation for Dr. Parentin is Institute for Maternal and Child Health - IRCCS "Boris Garofolo," Trieste, Italy. We regret the error.

doi:10.3899/jrheum.120583.CI 